\title{
Pattern of Hepatitis A Incidence According to Area Characteristics Using National Health Insurance Data
}

\author{
Joo Youn Seo ${ }^{1}$, Jae Hee Seo ${ }^{1}$, Myoung Hee Kim², Moran Ki ${ }^{3}$, Hee Suk Park ${ }^{1}$, Bo Youl Choi ${ }^{1}$ \\ 'Department of Preventive Medicine/Institute of Community Health, Hanyang University College of Medicine, Seoul; \\ ${ }^{2}$ People's Health Institute, Seoul; ${ }^{3}$ Department of Preventive Medicine, Eulji University School of Medicine, Daejeon, Korea
}

\begin{abstract}
Objectives: Over the past several years, the incidence of hepatitis A infection has been increasing rapidly in the youngadult population in Korea. We examined the effects of area-level socioeconomic status and environmental hygiene on the incidence of hepatitis $A$.

Methods: This study is based on the registered national population of Korea and the national health insurance data from 2004 to 2008. A total of 73459 individuals were confirmed to have had hepatitis A. The standardized incidences of hepatitis A in 232 districts adjusted for sex and age of people were calculated for each year, and the rate ratios of the incidence rates were estimated according to area-level socioeconomic status and environmental hygiene using multiple Poisson regression models.

Results: The incidence rates of hepatitis A infection were 15.6 (per 100 000) in 2004, 19.0 (per 100 000) in 2005, 27.2 (per 100000 ) in 2006, 25.1 (per 100 000) in 2007, and 61.7 (per 100000 ) in 2008. The analysis of the area-level effects showed that residential areas of the less deprived than other regions, areas with higher levels of education, and heavily populated areas were significantly associated with increased risk.

Conclusions: There is a very strong possibility that both area-level socioeconomic status and environmental hygiene play a role in increasing the risk of hepatitis A infection in Korea. Therefore, to reduce hepatitis A infection, we need a nationwide strategy that considers these area-level characteristics.
\end{abstract}

Key words: Hepatitis A, Korea, Population characteristics, Risk factors

J Prev Med Public Health 2012;45(3):164-173

\section{INTRODUCTION}

Acute hepatitis A is the most common cause of acute viral infection, and the age group that is primarily affected is 20- to 30-year-old adults [1]. In hepatitis A, which appears as an inapparent infection in cases where the original infection occurred during infancy, if the antibody retention rate decreases among infants, due to improvements in their living conditions and hygiene practice, there is a paradoxical tendency to have an increased risk, at initial presentation, of hepatitis A during adulthood [2]. Assessing the epidemiological situation, the antibody cultivation rate was more than 50\% until the 1980s. However, after the middle of the 1990s, the antibody cultivation rate decreased to less than $20 \%$, and, in the past ten years, there has been an increasing tendency for the disease to spread among adults, who are easily exposed to the hepatitis A virus [3-6]. When considering that the age group most prone to being infected by the hepatitis A virus is 20- to 30-year-old

(c) This is an Open Access article distributed under the terms of the Creative Commons Attribution Non-Commercial License (http://creativecommons.org/licenses/by-nc/3.0/) which permits unrestricted non-commercial use, distribution, and reproduction in any medium, provided the original work is properly cited. adults, who tend to have very active social lives, infection is not only a burden to society because of the direct medical expense, but also because of work absences or retirements that incur indirect social expenses [1,7].

It is known that hepatitis A is related to the socioeconomic status and environmental hygiene of a country [8]. First, in undeveloped regions, such as Africa, the Middle East, Central America, and some parts of Asia, that are categorized as highly dangerous [6,8-10], most hepatitis A infections occur during infancy, and the antibody cultivation rate is above $90 \%$. Second, developing countries and regions, such as Korea, Eastern Europe, and Russia, are considered intermediately dangerous [6,8-10] because the antibody cultivation rate is above $50 \%$, but among children ten years or younger, the rate is less than $20 \%$. In regions with intermediate danger, due to the amelioration of the socioeconomic status and environmental hygiene, the rate of hepatitis A infection in children is not high, but when a group is infected, it has a tendency to increasingly occur as an apparent infection among adults aged over 20. Finally,

Corresponding author : Bo Youl Choi, MD, PhD

222 Wangsimni-ro, Seongdong-gu, Seoul 133-791, Korea

Tel: + 82-2-2220-0662, Fax: + 82-2-2293-0660, E-mail: bychoi@hanyang.ac.kr

Received: 8 July 2011, Accepted: 10 October 2011 
developed countries and regions, such as Western Europe, Northern Europe, North America, and Japan, are categorized as not very dangerous [6,8-10], but even within these regions and countries, in the areas where the socioeconomic status is low, the antibody cultivation rate is low; for those in groups that tend to have high infection rates, such as illicit drug users, men who have sex with men, travelers, and medical professionals, inoculation is strongly recommended $[6,9,10]$. Likewise, the epidemiology of hepatitis A appears differently according to socioeconomic status and environmental hygiene, even within a single county $[6,10,11]$.

Outbreaks of hepatitis A have been increasing over the last ten years, alongside the improvement of socioeconomic conditions in Korea, and despite the fact that there are huge disparities in socioeconomic status among the regions, epidemiological studies targeting hepatitis A in Korea are rare [5]. Many studies in Korea have explored the correlation between residential location and hepatitis A incidence, but they have not been able to find any correlation between the socioeconomic index, the hygiene standards, and the incidence, and the targeted research region was confined only to the capital of Korea [12-14]. Thus, insufficient attention has been paid to a possible correlation between the incidence of hepatitis A and geographical location. Korea is a country with homogeneous social and housing conditions and a frequent exchange of travelers among regions, but hepatitis A incidence varies in the different regions. Therefore, grasping the primary factors that cause these huge regional differences in the hepatitis A incidence rate has public health implications [2,5]. In cases where hepatitis A infection continues susceptible residents, who live in regions where the antibody retention rate is low, the public health implications are even greater, as the hepatitis A infection can spread and can become a major disease burden on the residents.

By using National Health Insurance data, which is a representative source of data on Koreans, and by targeting subjects infected with hepatitis A, the authors of this calculated the incidence of hepatitis A in each city, district, and borough (si, gun, and gu), and studied the relevance of socioeconomic status and environmental hygiene to hepatitis A outbreaks.

\section{METHODS}

\section{Data and Subjects}

The incidence rate of hepatitis A was calculated based on liver disorder check-up bill data, as this was the main source, collected by the National Health Insurance Corporation from January of 2004 until December of 2008, and using Statistics Korea's Resident Registration Population Statistics data and Population Residence Census data for 2005. From the liver disorder check-up bill data, the patients with one or more hepatitis A-related disease categorization codes were included. The hepatitis A disease categorization codes include the International Classification of Diseases 10 classification system, and the related disease codes include B15 (acute hepatitis A), B15.0 (hepatitis A with hepatic coma), and B15.9 (hepatitis A without hepatic coma). By using these disease classification codes, a total of 168522 cases of check-up bill data were confirmed; after overlapping cases were consolidated, 95063 incidences and 72941 people (518 people excluded because their residence address data was missing) were included in the study as targets who had contracted hepatitis A.

In addition, the statistical year book produced by the association of local governments, the Korean Statistical Information Service (KOSIS) data, and the Population Residence Census data of 2005 were used to determine regional socioeconomic status and environmental hygiene.

In order to calculate the incidence rate of hepatitis A at the city, district, and borough levels using population data, the Resident Registration Population Statistics data was used. In order to suggest a standardized incidence rate, an administration district was unified for five years, and a total of 232 sites were categorized based on the administration district. Since the sex and age population structure by city, district, and borough was found to be different, a standardized incidence was calculated through direct standardization, and the entire Korean population of the Population Residence Census was used in order to determine the average period of time for the population.

The socioeconomic status and the environmental hygiene were determined from the statistical year book for 2008 (compiled by an association of local governments), the KOSIS National Statistics Portal's statistical data by topic, and the Region Deprivation Index and residence concentration levels applied to the Population Residence Census and Population Residence Census 2\% sample survey of 2005 [15]. The indicators used from each archive are listed below [15-17].

\section{Variables}

\section{A. Dependent variable}

In this study, after having standardized sex and age, hepatitis A incidence rates were calculated (per 100000 
people) according to city, district, and borough sites $(n=232)$, and we attached this data and the data on socioeconomic status and environmental hygiene levels. The incidence rate was directly standardized by dividing the population by age, in groups of ten years, and by sex, and the standardized incidence rate became a dependent variable. Due to the fact that the National Health Insurance Corporation source only includes data on hepatitis A patients, in order to calculate the standardized incidence rate, we added up the population numbers, according to sex, age, and region, to form the denominator of the incidence rate. After calculating the crude incidence rate, using the Population Residence Census of 2005 as the standard population, a total of 1160 standardized incidence rates for different subsections of the population were calculated for the sex and ten-year age groups and for 232 separate sites.

\section{B. Independent variable}

Independent variables contained in the socioeconomic status index included the proportion of those receiving assistance for basic living expenses (\%), taken from the statistical year book for 2008; the self-reliance ratio of local finance $(\%)$, taken from the Korean statistical information service for 2008; and the proportion of the heads of household who were high school graduates $(\%)$, taken from the 2005 census [18].

In addition, the Korean regional deprivation index was used. This deprivation index includes the falling behind residence environment ratio the elderly population ratio, the proportion of the population who have not graduated from high school, the low social class ratio, the proportion of apartment-living households, the proportion of households without a car, the proportion of single households, and the proportion of female-headed households. The definition of "fallen-behind living environment" is a residence "without a separate kitchen, no water supply facilities, no hot water bath, and no flush toilet" [15]. It is also defined as a region where the higher the regional deprivation index level, the more fallen- behind the living environment.

As for the environmental hygiene index (using the 2008 standard), the water supply rate, which is the population density (person $/ \mathrm{km}^{2}$ ) divided by the residence density (residence registration population numbers per area $\left[\mathrm{km}^{2}\right]$ ), represents household overcrowding (the ratio that represents a residential area that is occupied by more than 1.5 people out of many residents), and this was used to calculate the environmental hygiene index. Every variable was classified into a high, medium, or low level. Table 1 shows the types of independent variables and raw data sources.

\section{Methods of Analysis}

In this study, the incidence rates of 2008 were mainly used and described in Table 2 and 3 in detail. Not only were there timely index of coincidence values the highest, but they had the closest agreement to the hepatitis A sample surveillance data from the Korea Centers for Disease Control and Prevention (although the latter was not used for statistical analysis in this study) [19]. In order to determine the difference in the appearance of hepatitis A according to region, this study described that the incidence rate of the entire nation's 16 metropolitan cities and provinces, and the incidence rate according to the distribution of independent variables using analysis of variance. Furthermore, the study suggested that the occurrence appearance was related to regions and age groups, and in order to explore the relationship between the independent variables and the incidence rate, the rate ratio (RR) and $95 \%$ confidence interval (CI) were calculated using multivariate Poisson regression analysis, Multivariate Poisson regression analysis is a statistical analysis method that is applied to obtain, as a result variable, the incidence rate of a disease that is rare in a large population group [20]. Those receiving assistance for basic life expenses and those who had graduate from high school were highly correlated with the deprivation index; thus, these two variables were excluded from this

Table 1. The data sources of area-level socioeconomic and environmental characteristics of 232 districts, Korea

\begin{tabular}{|c|c|c|}
\hline & Parameter & Data source \\
\hline \multirow[t]{4}{*}{ Socioeconomic factor } & Deprivation index ${ }^{1}$ & Census, 2005 \\
\hline & The proportion of those receiving assistance for basic living expenses (\%) & Statistical year book, 2008 \\
\hline & High school graduate head of household (\%) & Census, 2005 \\
\hline & Self-reliance ratio of local finance (\%) & Korean Statistical Information Service, 2008 \\
\hline \multirow[t]{3}{*}{ Environmental factor } & Population density $\left(/ \mathrm{km}^{2}\right)$ & Statistical year book, 2008 \\
\hline & Household overcrowding ${ }^{2}$ & Census, 2005 \\
\hline & Water supply (\%) & Korean Statistical Information Service, 2007 \\
\hline
\end{tabular}

${ }^{1}$ Increasing deprivation indices, the districts are more deprived areas.

${ }^{2}$ Household overcrowding: the proportion of members of a household in a crowded condition ( $>1.5$ persons/room) in an area. 
analysis. Due to the fact that the ratio of those who were high school graduates or higher (which shows the education level of the subjects) was a lower variable of the deprivation index, the two variables show similar results to the deprivation index. Therefore, in this study, the deprivation index and the self-reliance ratio of regions were included in the analysis. Model I of Table 4 used only the socioeconomic status variables, while Model II was analyzed after adding the environmental hygiene variables. In order to visualize the incidence rate, we manifested the standardized incidence rate as a map using a geographic information system. For want of space, only the map of 2008 with the highest incidence rate is presented here. All statistical analysis was performed using SAS version 9.2 (SAS Inc., Cary, NC, USA), and the map was drawn using the geographic information program MapWizard for Excel 2007 (Tastech Inc., Seoul, Korea).

\section{RESULT}

\section{Incidence Aspect of National Hepatitis A}

The incidence rate of hepatitis A (per 100000) was 14.3 in 2004, 16.1 in 2005, 22.2 in 2006, 21.5 in 2007, and 51.5 in 2008; this shows a slow rise, followed by a rapid increase in 2008. In order to visualize the incidence of hepatitis A, the incidence rate of hepatitis A for each location was marked on the map. For want of space, we include only the national unit map and the map of the Seoul metropolitan area (Figure 1). The national distribution shows a relatively high incidence rate in the capital area, especially in the southwest of the metropolitan area. On the other hand, Korea's eastern inland shows a low incidence rate, and the southeastern province of Gyeongbuk has a particularly low incidence rate. When considering the Seoul area's incidence rate per borough, the northern part of Seoul, which has a high deprivation rate, has a low socioeconomic status level and a high incidence rate, and in the southern part of Seoul, a low deprivation rate with a low incidence rate was observed. The average incidence rates in 2008 for the 232 city, district, and borough sites, as well as the independent variable, are shown in Table 2. Based on 2008 , the average incidence rate of subjects in Korea infected with hepatitis A was 51.5 people, and the lowest sites had an incidence rate of 0 per 100000 (Ulleung-gun, Yeongyang-gun of Gyeongbuk province, southeast inland region and islands of Korea), and the site with the highest incidence had an incidence rate of 169.1 people (Incheon Dong-gu metropolitan area and its vicinity, near the coastal area).

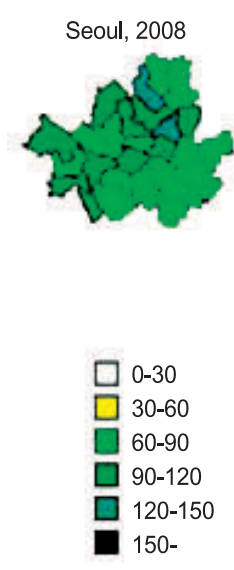

Figure 1. The 2008 Seoul and nationwide of Korea distribution of hepatitis A incidence (/100 000) and number of districts with each incidence level.

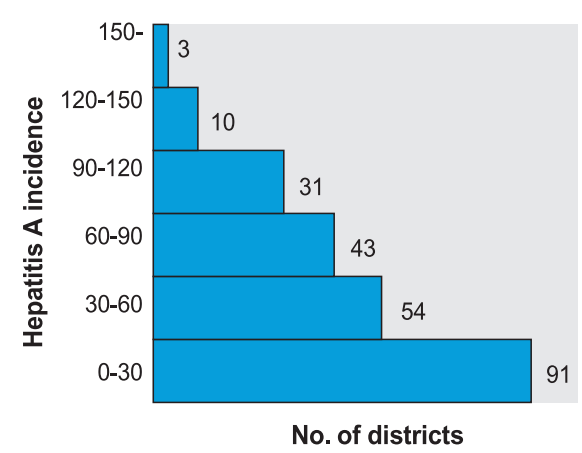


Table 2. The distribution of hepatitis A incidence (/100000) in 2008 and area-level socioeconomic and environmental characteristics of 232 districts, Korea

\begin{tabular}{lcccccccc}
\hline \multicolumn{1}{c}{ Parameter } & Mean & SD & Minimum $\begin{array}{c}\text { 25th } \\
\text { percentile }\end{array}$ & Median & $\begin{array}{c}75 \text { th } \\
\text { percentile }\end{array}$ & Maximum & IQR \\
\hline Hepatitis A incidence (/100 000), 2008 & 51.5 & 37.3 & 0 & 19.6 & 41.4 & 80.2 & 169.1 & 60.5 \\
\hline Socioeconomic factors & & & & & & & \\
$\quad$ Deprivation index & 0.06 & 0.90 & -1.47 & -0.71 & -0.07 & 0.80 & 1.70 & 1.51 \\
$\quad$ The proportion of those receiving assistance for basic living expenses (\%) & 4.4 & 2.2 & 0.7 & 2.4 & 4 & 5.9 & 11.3 & 3.5 \\
$\quad$ High school graduate head of household (\%) & 58.2 & 19 & 21.1 & 41.7 & 63.4 & 74.5 & 92.3 & 32.8 \\
$\quad$ Self-reliance ratio of local finance (\%) & 65.9 & 10.1 & 36.4 & 60.9 & 66.9 & 72.4 & 90.4 & 11.5 \\
Environmental factors & & & & & & & & \\
$\quad$ Population density (/km²) & 4010 & 6391 & 19.2 & 101 & 408 & 5895 & 28870 & 5794 \\
$\quad$ Household overcrowding & -0.008 & 1 & -3.55 & -0.55 & -0.04 & 0.59 & 3.43 & 1.1 \\
$\quad$ Water supply (\%) & 82 & 20.3 & 28.4 & 66.5 & 90.9 & 99.7 & 100 & 34.2 \\
\hline
\end{tabular}

SD, standard deviation; IQR, interquartile range.

${ }^{1}$ Household overcrowding: the proportion of members of a household in a crowded condition (>1.5 persons/room) in an area.

\section{Incidence Rate of Hepatitis A According to Regional Socioeconomic Level and Environment}

The incidence rates, according to the socioeconomic status and environmental hygiene levels within the 232 city, district, and borough sites, are shown in Table 3. According to the socioeconomic status level of each region, a lower regional deprivation rate, a lower proportion of those receiving assistance for basic life expenses, and a higher percentage of the population with an education level above high school were related to a higher incidence rate. A high incidence rate was found in the regions with the highest third of the self-reliance ratios, while the regions in the lower and middle thirds showed a similar incidence rate. In particular, variables that showed a distinct tendency were the deprivation index, the proportion of those receiving assistance for basic life expenses, and the education level. The incidence rate for regions in the lower third by deprivation rate (i.e., regions with lower deprivation) was 62.8 (per 100000), while that of regions in the higher third was 38.0. Regions ranking in the bottom third for those receiving assistance for basic life expenses had an incidence of 71.5 ; regions in the top third had an incidence of 43.7. Regarding education level, the region with the lowest high school graduation rate had an incidence of 38.1, while the region with the highest high school graduation rate had an incidence of 69.2, and this shows that differences among socioeconomic status index levels are related to the differences in the incidence levels, aside from other environmental hygiene indexes, with the exception of population density.

As for the environmental hygiene index, regions with a high population density, with a high water supply
Table 3. Nationwide sex- and age-standardized hepatitis $A$ incidences $(/ 100000)$ according to the level of socioeconomic and environmental hygiene factors of districts, South Korea, 2008

\begin{tabular}{|c|c|c|}
\hline Parameters & $\begin{array}{c}\text { Hepatitis } \\
\text { A incidence }^{1}, 2008\end{array}$ & $p$-value ${ }^{4}$ \\
\hline \multicolumn{3}{|l|}{ Socioeconomic factors } \\
\hline \multicolumn{3}{|l|}{ Deprivation index ${ }^{2}$} \\
\hline High & 38.0 & $<0.001$ \\
\hline Medium & 54.3 & \\
\hline Low & 62.8 & \\
\hline \multicolumn{3}{|c|}{$\begin{array}{l}\text { The proportion of those receiving } \\
\text { assistance for basic living expenses (\%) }\end{array}$} \\
\hline High & 43.7 & $<0.001$ \\
\hline Medium & 41.6 & \\
\hline Low & 71.5 & \\
\hline \multicolumn{3}{|c|}{$\begin{array}{l}\text { High school graduate head of } \\
\text { household (\%) }\end{array}$} \\
\hline Low & 38.1 & $<0.001$ \\
\hline Medium & 42.6 & \\
\hline High & 69.2 & \\
\hline \multicolumn{3}{|c|}{ Self-reliance ratio of local finance (\%) } \\
\hline Low & 47.1 & $<0.001$ \\
\hline Medium & 42.8 & \\
\hline High & 61.5 & \\
\hline \multicolumn{3}{|l|}{ Environmental factors } \\
\hline \multicolumn{3}{|l|}{ Population density $\left(/ \mathrm{km}^{2}\right)$} \\
\hline Low & 40.9 & $<0.001$ \\
\hline Medium & 52.7 & \\
\hline High & 66.7 & \\
\hline \multicolumn{3}{|l|}{ Household overcrowding ${ }^{3}$} \\
\hline Low & 43.5 & 0.016 \\
\hline Medium & 54.0 & \\
\hline High & 55.2 & \\
\hline \multicolumn{3}{|l|}{ Water supply (\%) } \\
\hline High & 55.6 & 0.001 \\
\hline Medium & 56.9 & \\
\hline Low & 41.4 & \\
\hline
\end{tabular}

${ }^{1}$ Sex- and age-standardized hepatitis A incidences by direct standardization using the census, 2005.

${ }^{2}$ Increasing deprivation indices represent the districts that are more deprived.

${ }^{3}$ Household overcrowding: the proportion of members of a household in a crowded condition (>1.5 persons/room) in an area.

${ }^{4} p$-value by ANOVA test. 
Table 4. The nationwide association between socioeconomic and environmental factors of districts and sex- and age-standardized hepatitis A incidences from multiple Poisson regression models, Korea, 2008

\begin{tabular}{|c|c|c|}
\hline \multirow{2}{*}{ Parameters } & Model I & Model II \\
\hline & $\mathrm{RR}(95 \% \mathrm{Cl})$ & $\mathrm{RR}(95 \% \mathrm{Cl})$ \\
\hline $\begin{array}{l}\text { Incidence of } \\
\text { previous year } \\
(2007)\end{array}$ & 1.00 & 1.00 \\
\hline \multicolumn{3}{|l|}{ Socioeconomic } \\
\hline \multicolumn{3}{|l|}{$\begin{array}{l}\text { factors } \\
\text { Deprivation index }\end{array}$} \\
\hline High & Reference & Reference \\
\hline Medium & $1.26(1.26,1.26)$ & $1.17(1.17,1.17)$ \\
\hline Low & $1.35(1.35,1.35)$ & $1.29(1.29,1.29)$ \\
\hline \multicolumn{3}{|l|}{$\begin{array}{l}\text { Self-reliance ratio } \\
\text { of local finance }(\%)\end{array}$} \\
\hline Low & Reference & Reference \\
\hline Medium & $0.97(0.97,0.97)$ & $0.94(0.94,0.94)$ \\
\hline High & $1.11(1.11,1.11)$ & $1.16(1.16,1.16)$ \\
\hline \multicolumn{3}{|l|}{ Environmental } \\
\hline \multicolumn{3}{|l|}{$\begin{array}{l}\text { Population } \\
\text { density }\left(/ \mathrm{km}^{2}\right)\end{array}$} \\
\hline Low & & Reference \\
\hline Medium & & $0.95(0.95,0.95)$ \\
\hline High & & $1.38(1.38,1.38)$ \\
\hline \multicolumn{3}{|l|}{ Household } \\
\hline Low & & Reference \\
\hline Medium & & $1.17(1.17,1.17)$ \\
\hline High & & $1.25(1.25,1.25)$ \\
\hline \multicolumn{3}{|l|}{ Water supply (\%) } \\
\hline High & & Reference \\
\hline Medium & & $1.27(1.27,1.27)$ \\
\hline Low & & $1.32(1.32,1.32)$ \\
\hline
\end{tabular}

$\mathrm{RR}$, rate ratio; $\mathrm{Cl}$, confidence interval

${ }^{1}$ Increasing deprivation indices represent the districts that are more deprived.

${ }^{2}$ Household overcrowding: the proportion of members of a household in a crowded condition ( $>1.5$ persons/room) in an area.

distribution rate, or with highly overcrowded households showed a high incidence rate. However, this was a result of examining only one index, and the results for various indexes together are shown in Table 4.

Hepatitis A can be spread through person-to-person contact; thus, the incidence rate of former years is suggested as a confounding variable. Model I shows that, when the higher third is treated as a reference, the $\mathrm{RR}$ in the regions in the middle third is 1.26 (95\% CI, 1.26 to 1.26$)$, and that of the lower-third regions is 1.35 (95\% CI, 1.35 to 1.35 ), which demonstrates that the lower the deprivation rate, the higher the incidence rate. On the other hand, the incidence rate had no consistent trends relative to the self-reliance ratio. In Model II, as a result of having added the environmental hygiene index to the analysis, the higher the population density, the

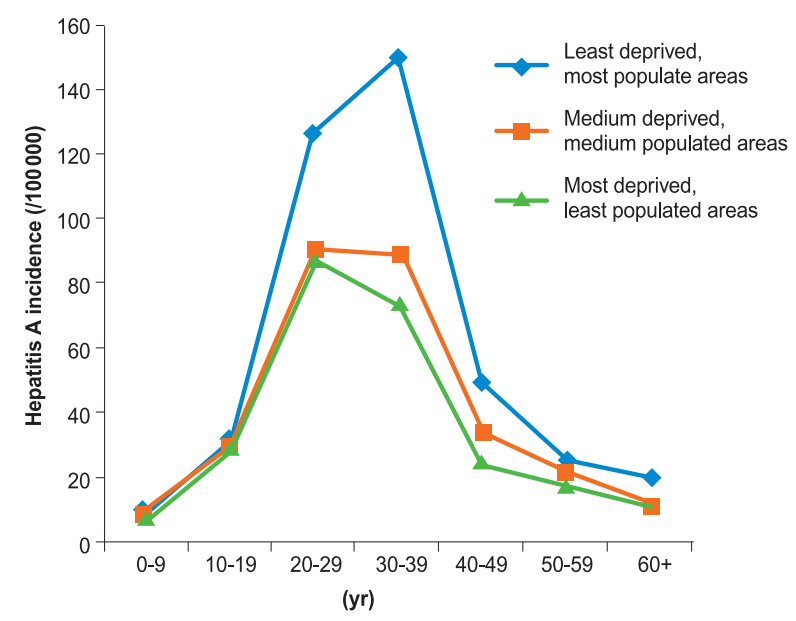

Figure 2. The 2008 nationwide of Korea hepatitis A incidence (/100000) according to deprivation index and population density of area.

higher the incidence rate, as the higher residence concentration indicates household overcrowding. Compared to the regions in the lowest third for population density, those in the top third had an RR of 1.38 (95\% CI, 1.38 to 1.38 ). The correlation coefficient of the regional deprivation index and the proportion of those receiving assistance for basic life expenses was 0.82 , while the correlation coefficient of high school graduates was -0.96 , and these two variables were excluded in this analysis because the results were similar to those of the deprivation index analysis (except for some problems with multi-collinear characteristics).

When looking at the incidence rate per age group, according to regional characteristics, as a whole the region that had the lowest deprivation index with the highest population had the highest incidence rate, but this depended on age group. The region where the deprivation index was the highest and the population was low (i.e., a "fallen-behind environment") had the highest incidence rate among people in their 20s. The regions ranking in the middle third on the deprivation index and for population density showed similar incidence rates among those in their 20 s and 30 s. In the region where urbanization involved the lowest deprivation rate and a high population density, the highest incidence was in those in their 30s (Figure 2).

\section{DISCUSSION}

This study divided the Republic of Korea into 232 city, district, and borough sites, obtained the incidence rate of each region, and studied the correlation between the 
region's socioeconomic and environmental hygiene indexes and the incidence rate. The National Health Insurance data used in this study was appropriate for descriptive epidemiology, including time series study, as it is known to be a source that has a distribution similar to that of the actual patient distribution [21].

This carefully examined other studies that showed a strong relationship between hepatitis $\mathrm{A}$ and regional characteristics, both inside and outside Korea. In Brazil, for example, the higher the education level, the water supply rate, and the solid trash pick-up rate by region, the lower the antibody cultivation rate, and this connection was statistically significant. Furthermore, in urbanizing regions, the state of public hygiene, such as the water supply rate, is a very strong risk factor for hepatitis A infection [11]. In Taiwan, for example, the antibody cultivation rate was high in regions where the people drank dirty (non-tap) water [22]. Moreover, among the 157 countries that have been studied, in studies where the authors were exploring the relationship between socioeconomic variables, including the water supply rate, the life expectancy, the gross domestic product, the education level, and the hepatitis A antibody cultivation rate, most of the variables were found to be significantly related. In addition, a strong negative correlation was found between the water supply rate and the antibody cultivation rate [8]. According to research reported in Korea in 2007 [10], the antibody cultivation rate was higher in rural areas than urban areas such as Seoul, especially among those under 40 years of age in Seoul; it was also significantly higher in the poorer regions than in the richer regions. Furthermore, hepatitis $\mathrm{A}$ is centered around Seoul and other big cities, and is concentrated in metropolitan areas [4]. We analyzed the incidence rate by region in order to identify outbreaks of hepatitis $\mathrm{A}$ in Korea, from 2004 to 2008, to verify the hypothesis that hepatitis $\mathrm{A}$ is related to the socioeconomic and environmental hygiene levels of a region. We found that, in Korea, hepatitis A increased after 2004, and increased sharply in 2008, and that hepatitis A infection often occurs among young people in their 20s and 30s; these results agree with the existing study results $[1,7,23]$. Therefore, Korea can be regarded as an intermediately dangerous region for hepatitis A infection [4-6,9,10,24]. At the same time, except for some regions with disease epidemics, a high regional similarity exists in incidence every year. Because of environmental hygiene, the more a region's socioeconomic level, population density, and residence concentration (which results in more frequent contact among people) increased, the higher the incidence rate. In metropolitan areas, including Seoul and
Incheon, there was a relatively high incidence rate. In addition, when looking at regional distribution in Korea, Korea's southwestern region had a higher infection rate than the other regions, which implies a regional epidemic of hepatitis A.

Hepatitis A, in a country like Korea with an intermediate danger of infection, the socioeconomic level and the antibody cultivation rate are inversely proportional [11]. Furthermore, in a country like Korea, hepatitis A epidemics take place in regions with high socioeconomic status, where susceptible individuals are widely distributed. Korea was not equipped with water sewage systems until the 1980 s, which led to the insufficient provision of drinking water, and because much of the farmland used feces as a fertilizer until the 1980 s, most of the population was infected with inapparent hepatitis A in childhood via the fecal-oral route [2]. After improving the hygiene conditions in the country, few adults who were in their infancy after the 1980s were exposed to the disease, and thus there are many adults who now susceptible to the disease. Therefore, after the 1980 s, the way that people were exposed to hepatitis A infection changed. In other words, after the 1980s, it is assumed that the population group who spent their infancy in the city, where the socioeconomic level had greatly improved, probably had a lower chance of exposure to inapparent infection than the population group who spent their childhood in the countryside, where socioeconomic development was slow.

The results of the analysis in this study show that the lower the regional deprivation index, and the higher the educational level of the population, the greater the incidence rate of hepatitis $\mathrm{A}$. This means that the higher the socioeconomic level of a region, the more susceptible the adults in their 20 s and 30 s will be to hepatitis A infection, as they have vigorous social lives, with frequent contact with people from a wide geographic distribution. Although we made an effort to adjust the age groups through direct standardization, the reason why the incidence rate shows differences according to the age structure is because each region has a different herd immunity level. In other words, regions with high socioeconomic levels have low herd immunity and show a high incidence rate. In terms of environmental hygiene conditions, the incidence rate increased in regions where there was a high population density, the residents could not access clean water, and household overcrowding was high. Thus, there was a tendency for the incidence rate to increase as the region developed conditions for people to have frequent contact. On the other hand, when looking 
at incidence rate results within the city of Seoul in 2008 , there was a tendency for the incidence rate to increase where the deprivation index and socioeconomic levels were low. This is not the same as the results for the other regions of Korea, likely because in Seoul the socioeconomic level was low, the vaccination rate was low, and the hygiene conditions were not favorable. However, in this study, the authors could not confirm these results because there were not sufficient data to understand other hygiene conditions. Further study is needed in this regard.

In this study, the age group with the highest incidence rate varied according to the region: when the region was less developed, the incidence rate was at the highest level for those in their 20s, while in urbanized regions, the incidence rate was at the highest level for those in their 30s. The reason for this can be explained as follows: First, many of those in the 20s age cohort may actually reside in different regions than the ones stated on their social security cards. Many colleges in Korea are located in large cities where the deprivation rate is low. Thus, there may be a high ratio of college students in the 20 s age cohort who live in metropolitan cities, but graduated from high schools in cities that are relatively less developed or have fallen behind. There is a tendency for these students not to transfer their residence registration address before they get a stable job; thus, if many of those in their 20s, who used to live in farming and fishing villages, reside in metropolitan cities, then in reality the incidence rate, according to the deprivation rate, could be different than what the data reflects.

Second, in regions with a low deprivation rate, namely regions in which there is a low population of those in their 20s, there is a possibility that an epidemic of hepatitis A has occurred. In regions where the population is low, even if there is an epidemic among a small number of men, when calculating the incidence rate, there is a possibility that the incidence rate will increase exponentially.

Third, depending on the socioeconomic status and environmental hygiene level of a region, there are different age groups that contract hepatitis $\mathrm{A}$ and acquire the antibody. According to a study performed in Brazil, in which the danger level is intermediate [11], wherever the socioeconomic status varied, the antibody cultivation rate differed according to age group. In the most backward region, the Northeastern region, the hepatitis A antibody cultivation rate before the age of five was $31.5 \%$, and in the federal region, where it was most developed, the cultivation rate was only $20 \%$. However, when the population reaches 19 years old, both regions have a similar antibody cultivation rate of $70 \%$, and this shows that the more developed a region is, the higher the age group that acquires the hepatitis A antibody. Therefore, the age variation phenomenon exists according to the socioeconomic status of a region.

The results of this study are comparable to earlier studies. As in this study, previous research has shown that individuals living in regions with a high socioeconomic status have a lower possibility of inapparent infection during infanthood than those in regions with a low socioeconomic status. Nevertheless, a low education level and a low rate of water supply are understood as risk factors for hepatitis A infection [11]. In addition, the results of this study indicate that the contraction rate of hepatitis A increases with population density, which matches previous research results $[25,26]$.

There are a couple of limitations to this First, the incidence rate was calculated using only patients whose medical fees for hepatitis A infection were covered by the National Health Insurance Corporation, and thus it was impossible to understand the scale of the inapparent infection of hepatitis A. In reality, hepatitis A is easily ignored during infancy because it appears as an inapparent infection, and thus it is difficult to determine the number of childhood cases [23]. Second, when calculating the incidence rate, there is a possibility that the incidence rate could be greatly underestimated because the denominator includes people who already possess the antibody. Furthermore, in farming and fishing villages where the population was heavily weighted toward older people, such a tendency was intensified. Thus, with respect to the antibody cultivation rate for every region, underestimation of the incidence rate should be considered. Third, although there are different probabilities for being infected with hepatitis A according to an individual's education level and occupation [19], and although some previous studies have implemented multi-level analysis of individual and regional socioeconomic levels [11], this study failed to analyze the incidence aspect at an individual level due to the limited availability of data.

Despite its limitations, this study is significant for several reasons. First, this study has undertaken a process of targeting all citizens registered in the residence registration statistics, and this likely produced representative data that can reflect various aspects of hepatitis A incidence in Korea. Second, previous studies have targeted certain regions of Korea [12-14,24], but this study targeted the entire country. Third, this study analyzed not only the regional incidence rate itself, but also the relationship between the region's socioeconomic 
level and the incidence rate. In addition, this study is significant in the way that it explores the reasons for the regional differences in hepatitis $\mathrm{A}$ incidence. This study hows that there can be changes in the hepatitis $\mathrm{A}$ incidence rate related to the socioeconomic status and environmental hygiene of Korean regions. It is a very important public eubiotic problem that, in epidemics of hepatitis A in a country with intermediate danger such as Korea, the incidence rate is higher in regions with a low antibody cultivation rate.

There are different trends in Korea according to regional socioeconomic status. When a region is socioeconomically backward, it is close to the epidemiological concept of a highly dangerous region, where there is much inapparent infection at an early age; on the other hand, the more socioeconomically developed a region is, the older the age of the infection class. In the current epidemic period, many susceptible people from socioeconomically developed regions are infected. In order to overcome this and become a low danger country, not only is it necessary to have well-balanced development, but also aggressive prevention, which should be directed at young people who are susceptible and live in regions where there is a high danger of hepatitis A infection.

The primary methods for preventing hepatitis A infection are the improvement of public health conditions (in order to stop the spread of the hepatitis A virus), thorough individual hygiene maintenance, and hepatitis A vaccination [2]. Presently, hepatitis A vaccination is not a national mandatory vaccination in Korea, but rather an optional vaccination; adults over 40s are not recommended to receive vaccination. The government of strongly recommends the vaccination of young children up to 16 years old who do not have the hepatitis A virus antibody, and those in their 20s and 30s with hepatitis B, hepatitis $\mathrm{C}$, or orchronic epilepsy, as well as those who plan an extended stay in a hepatitis A-infected area, laboratory practitioners, and those who have come into contact with a hepatitis A-infected person within the previous two weeks [27].

However, as one can see from the present study, the epidemiological aspects of hepatitis A vary by region. Once an epidemic has begun, vaccination should be provided not only to those in highly endangered groups, but also to the general public in highly dangerous areas with low antibody cultivation rates, and the vaccination should be performed strategically.

Presently, the teenage population and those in their 20s have the lowest hepatitis A cultivation rate in Korea [7]. In the case of developed countries, considering the fact that it has taken ten years for the hepatitis A virus to be transferred to an intermediate epidemic trend region to low epidemic region [2], it can be predicted that there is a possibility that the number of adult and youth hepatitis Ainfected patients could grow rapidly grow. Based on these results, strategic vaccination is highly necessary, targeting youths and adults with high susceptibility. In reality, when looking at Israel, a country that is classified as an intermediate-danger region, after they performed a routine vaccination, where children between the ages of 1 and 2 were vaccinated, the number of patients with hepatitis A dramatically decreased [7,28,29]. However, considering the existing circumstances, in which a vaccination campaign targeting the whole nation is difficult due to high cost, it is very important to develop various strategies for vaccinating susceptible adults [2].

This study shows that differences in socioeconomic status can be a risk factor for hepatitis A infection. In case the hepatitis A infection epidemic trend continues, public health measures, such as strategic vaccination, ensuring a safe supply of drinking water, and being responsible for the clean handling of food ingredients, can be utilized.

\section{CONFLICT OF INTEREST}

The authors have no conflicts of interest with the material presented in this paper.

\section{REFERENCES}

1. Kang HM, Jeong SH, Kim JW, Lee D, Choi CK, Park YS, et al. Recent etiology and clinical features of acute viral hepatitis in a single center of Korea. Korean J Hepatol 2007;13(4):495-502 (Korean).

2. Park SH. Hepatitis A: past and present. J Korean Med Assoc 2009;52(10):996-1004 (Korean).

3. Eun JR, Lee HJ, Kim TN, Jang BI, Moon HJ. Clinical characteristics of adult patients with acute hepatitis A. Yeungnam Univ J Med 2007;24(2):170-178 (Korean).

4. Jung YK, Kim JH. Epidemiology and clinical features of acute hepatitis A: from the domestic perspective. Korean $\mathrm{J}$ Hepatol 2009;15(4):438-445 (Korean).

5. Kim JH. Recent epidemiological status and vaccination of hepatitis A in Korea. J Korean Med Assoc 2008;51(2):110118 (Korean).

6. Park JH. Changes in the seroprevalence of hepatitis A virus antibody in Korea. Korean J Hepatol 2007;13(1):1-4 (Korean).

7. Jeong SH. Current status and vaccine indication for hepatitis A virus infection in Korea. Korean J Gastroenterol 
2008;51(6):331-337 (Korean).

8. Jacobsen KH, Koopman JS. The effects of socioeconomic development on worldwide hepatitis A virus seroprevalence patterns. Int J Epidemiol 2005;34(3):600-609.

9. Jacobsen KH, Koopman JS. Declining hepatitis A seroprevalence: a global review and analysis. Epidemiol Infect 2004;132(6):1005-1022.

10. Song YB, Lee JH, Choi MS, Koh KC, Paik SW, Yoo BC, et al. The age-specific seroprevalence of hepatitis A virus antibody in Korea. Korean J Hepatol 2007;13(1):27-33 (Korean).

11. De Alencar Ximenes RA, Martelli CM, Merchan-Hamann E, Montarroyos UR, Braga MC, de Lima ML, et al. Multilevel analysis of hepatitis A infection in children and adolescents: a household survey in the Northeast and Central-west regions of Brazil. Int $\mathrm{J}$ Epidemiol 2008;37(4):852-861.

12. Kim DK, Kim YD, Jang HO, Jo JR. Prevalence of hepatitis A IgG antibody in children and adolescents in Gyeonggi province. Inje Med J 2006;27:53-58 (Korean).

13. Kim TY, Sohn JH, Ahn SB, Son BK, Lee HL, Eun CS, et al. Comparison of recent IgG anti-HAV prevalence between two hospitals in Seoul and Gyeonggi area. Korean J Hepatol 2007;13(3):363-369 (Korean).

14. Choi W, Eom HS, Kim IH, Lee DH, Kim PS, Kim HG, et al. Patterns of acute hepatitis A and anti-HAV seroprevalence of Kyungin province. Korean J Gastroenterol 1999;34(1):69-75 (Korean).

15. Management Center for Health Promotion, Hanyang University. Health promotion strategies and programmes development for health inequalities alleviation. Seoul: Ministry of Health \& Welfare; 2009, p. 227-303 (Korean).

16. Korea Statistical Information Service. Domestic statistics classified by subjects [cited 2012 Apr 30]. Available from: http://kosis.kr/abroad/abroad_01List.jsp (Korean).

17. Ministry of Public Administration and Security. Statistical year book of local government 2008. Seoul: Ministry of Public Administration and Security; 2009 (Korean).

18. Han JY, Na BJ, Lee MS, Hong JY, Lim NG. The relationship between local fiscal indices and standardized mortality rate. Proceedings of the KAIS Spring Conference; 2010 May 28-29; Cheonan, Korea. Seoul: Korea Academia-Industrial Cooperation Society; 2010, p. 1072-
1076 (Korean).

19. Ki MR, Choi BY. Economic analysis of vaccination and development of guidelines for control of hepatitis A virus infection, and epidemiologic analysis and development of control strategy of hepatitis C virus infection. Seoul: Korea Centers for Disease Control and Prevention; 2009, p. 38-41 (Korean).

20. Szklo M, Nieto FJ. Stratification and adjustment: multivariate analysis in epidemiology. In: Szklo M, Nieto FJ. Epidemiology: beyond the basics. 2nd ed. Sudbury: Jones and Bartlett Publishers; 2007, p. 246-281.

21. Park SK, Cheong HK, Ki M, Son YM, Kim H. Assessment of the availability of health insurance data for epidemiologic study of childhood aseptic meningitis. Korean J Prev Med 2003;36(4):349-358 (Korean).

22. Tsai CF, Lin DB, Chen SC, Chang YH, Chen CY, Lin JB. Seroepidemiology of hepatitis A virus infection among schoolchildren in Taiwan. J Med Virol 2011;83(2):196-200.

23. Choi HJ, Lee SY, Ma SH, Kim JH, Hur JH, Kang JH. Age related prevalence of antibodies to hepatitis A virus: performed in Korea in 2005. Korean J Pediatr Infect Dis 2005;12(2):186-194 (Korean).

24. Kwon YO, Choi IJ, Jung JW, Park JH. An epidemiologic study on the seropositive rate of hepatitis A virus among a selected group of children and adults in Busan. Korean $\mathrm{J}$ Pediatr 2007;50(3):262-267 (Korean).

25. Venczel L, Brown S, Frumkin H, Simmonds-Diaz J, Deitchman S, Bell BP. Prevalence of hepatitis A virus infection among sewage workers in Georgia. Am J Ind Med 2003;43(2):172-178.

26. Tosti ME, Spada E, Romano L, Zanetti A, Mele A; SEIEVA collaborating group. Acute hepatitis A in Italy: incidence, risk factors and preventive measures. J Viral Hepat 2008;15(Suppl) 2:26-32.

27. Korea Centers for Disease Control and Prevention. Epidemiology and control of vaccination targeted infectious disease. Cheongwon: Korea Centers for Disease Control and Prevention; 2010, p. 341-349 (Korean).

28. Koslap-Petraco MB, Shub M, Judelsohn R. Hepatitis A: disease burden and current childhood vaccination strategies in the United States. J Pediatr Health Care 2008;22(1):3-11.

29. Chodick G, Shalev V. Declining incidence of hepatitis A. JAMA 2006;295(3):282. 